\title{
Effect of Tarantula Cubensis Extract (Theranekron) on Peripheral Nerve Healing in an Experimental Sciatic Nerve Injury Model in Rats
}

\author{
Zahir KIZILAY ${ }^{1}$, Serdar AKTAS ${ }^{2}$, Nesibe KAHRAMAN CETIN³ ${ }^{3}$, Mahmut Alp KILIC ${ }^{4}$, Hakan OZTURK \\ ${ }^{1}$ Aydin Adnan Menderes University, School of Medicine, Department of Neurosurgery, Aydin, Turkey \\ ${ }^{2}$ Aydin Adnan Menderes University, School of Medicine, Department of Pharmacology and Toxicology, Aydin, Turkey \\ ${ }^{3}$ Aydin Adnan Menderes University, School of Medicine, Department of Pathology, Aydin, Turkey \\ ${ }^{4}$ Aydin Adnan Menderes University, School of Medicine, Department of Physiology, Aydin, Turkey \\ ${ }^{5}$ Aydin Adnan Menderes University, School of Medicine, Department of Biostatistics, Aydin, Turkey \\ Corresponding author: Zahir KIZILAY zahir.kizilay@adu.edu.tr
}

\section{ABSTRACT}

AIM: To investigate the effects of systemic application of Theranekron on peripheral nerve healing after compression type peripheral nerve injury.

MATERIAL and METHODS: Twenty-one female Wistar albino rats were randomly divided into 3 groups ( $n=7)$ : Control (C), injury (I), and Theranekron $(T)$. The right sciatic nerves of rats in the I and T groups were compressed via an aneurysm clip for 5 minutes and $0.3 \mathrm{ml}$ Theranekron D6 was applied via subcutaneous administration once a week in the T group for a total period of four weeks. Nerve conduction velocity and proximal and distal latency of the rats were measured at the end of day 30 . The right sciatic nerves of the rats were then removed and myelin damage grading, axon counting, fibrosis assessment, caspase-3, and NF-kB immunochemical staining were performed. The data were analysed statistically and a $p$ value of less than 0.05 was considered to be significant.

RESULTS: Axonal degeneration, vacuolization and myelin destruction were found to be markedly greater in group T. Fibrosis and caspase-3 immunoreactivity were less intense in group T. There was a statistically significant difference in the electrophysiological results of groups I and T. However, there were no statistically significant differences in axon number and NF-kB immunochemical evaluation of groups I and $\mathrm{T}$.

CONCLUSION: The findings of this study show that Theranekron decreases axonal and myelin damage after sciatic nerve injury and that this neuroprotective effect of Theranekron can be attributed to its anti-inflammatory effect on pro-inflammatory cytokine levels.

KEYWORDS: Peripheral nerve, Injury, Tarantula cubensis extract, Theranekron, Healing, Rat

\section{INTRODUCTION}

$\mathrm{C}$ ellular and biochemical process such as neutrophil migration, accumulation of macrophages, biochemical agent release, Schwann cell proliferation, and protein synthesis occur after a traumatic injury to a peripheral nerve
(19). The aim of these cellular and biochemical processes is to repair the peripheral nerve and allow it to return to its original functional and structural state. However, proper regulation of pro-inflammatory cytokines and inflammatory cells requires healing of the injured peripheral nerve. An increasing number 
of studies have determined that pro-inflammatory cytokines such as tumor necrosis factor- $\alpha$ (TNF- $\alpha$ ) and interleukin- $1 \beta$ (IL-1B) can have a role in the mechanism of neuropathic pain $(11,16,23)$. In addition, research has also shown that using anti-inflammatory agents can support nerve regeneration and myelination $(9,13,15)$.

Theranekron is an alcohol extract of the Tarantula cubensis extract which is derived from the spider Tarantula cubensis (21). Limited studies have shown its beneficial effects in the treatment of bovine cutaneous papillomatosis, canine mammary adenocarcinomas, as well as in traumatic tendon injuries in rats, and in many inflammatory lesions, and wound healing processes $(1,6,7,17)$. Although the mechanisms associated with its beneficial effect have not been fully elucidated, some research has indicated that theranekron may have an anti-inflammatory and inhibitory effect on catabolic enzymes (8). However, no studies have shown the effect of the Tarantula cubensis extract on a traumatic peripheral nerve injury.

In this study, we investigated the effects of the Tarantula cubensis extract on peripheral nerve healing in a compression type peripheral nerve injury model and discussed the results in the light of the current literature.

\section{MATERIAL and METHODS}

After obtaining the necessary approval from the Aydin Adnan Menderes University Animal Ethics Committee (Approval Number: 64583101/2018/033), all experiments were performed according to the National Institutes of Health $(\mathrm{NIH})$ Guide for the Care and Use of Laboratory.

\section{Animals}

Twenty-one adult female Wistar albino rats weighing $250 \pm$ $10 \mathrm{~g}$ were used in this experimental study. All animals were housed under environmentally-controlled conditions at 20$24^{\circ} \mathrm{C}, 50 \pm 10 \%$ humidity, and a 12 -hour light/dark cycle. The rats were provided free access to food and water.

\section{Experimental Groups}

The rats were randomly divided into the three groups $(n=7$, each).

Group 1: Control (C); right sciatic nerve was exposed and closed.

Group 2: Injury (I); right sciatic nerve was exposed and a compression injury was induced via a FE-572K Yasargil aneurysm clip (Aesculap AG \& Co., Tutlingen, Germany) for five minutes. The aneurysm clip was then opened and the injured area was tied with 4-0 silk sutures and the layers were closed according to the original anatomical conformation.

Group 3: Theranekron (T); right sciatic nerve was exposed; a compression injury was performed, and the injured area was tied, and the anatomical layers were closed, respectively. Then, $0.3 \mathrm{ml}$ Theranekron D6 (Richter Pharma, Wels, Austria) were applied via subcutaneous administration per rat once a week for a total period of four weeks.

\section{Anaesthesia, surgical procedures and application of the Tarantula cubensis extract}

Fifty $\mathrm{mg} / \mathrm{kg}$ of ketamine (Ketasol, Richter Pharma, Austria) and $10 \mathrm{mg} / \mathrm{kg}$ of xylazine (BIOVETA PLC, Czech Republic) were used to anaesthetise each rat and the rats' body temperature was strictly monitored and maintained at $37^{\circ} \mathrm{C}$. The rats were placed on a table on prone position. Every phase of the operation was performed by the same surgeon using microsurgical techniques. The right gluteal region of the rats was shaved and wiped by using povidone-iodine (Batticon, Adeka Co., Samsun, Turkey). An oblique incision parallel to the right sciatic nerve was performed, and the biceps femoralis muscle was visualized. The right sciatic nerve was exposed after a blunt dissection of the biceps femoralis muscle. The right sciatic nerve compression in the I and $T$ groups was performed for five minutes using an FE-752K Yasargil aneurysm clip (12). Then, the aneurysm clip was opened and the injured area was tied with 4-0 silk sutures. The operation side was closed. After, $0.3 \mathrm{ml}$ of Theranekron D6 (Richter Pharma, Wels, Austria) were applied via subcutaneous administration in the $T$ group once a week for a total period of four weeks.

\section{Histopathological Evaluation}

Sciatic nerve samples were harvested from the proximal end to the bifurcation and placed in 10\% buffered formalin solution for pathological examination. The samples were taken for routine tissue processing after fixation. Then, they were left for 14 hours in the automated tissue processing device. The tissue samples were embedded in paraffin; the paraffin block was sectioned into 3-4 $\mu \mathrm{m}$ thick sections using a microtome. Slides prepared from the sections were stained with haematoxylin and eosin (H\&E), Toluidine blue and Masson's trichrome for routine histopathological examinations. Evaluation of axonal degeneration and fibrosis was conducted under 40x magnifications. Each section of the sciatic nerve was examined under a light microscope (Olympus BX52, Olympus Co., Tokyo, Japan). The routine sections were histologically graded for axonal changes and myelin disorganization (2). Swelling (pale staining) or shrinkage (dark staining) and vacuolization were observed in the axons due to degeneration. Myelin changes were typically seen, including attenuation, collapse or breakdown. Grading was performed on a scale of 0 to 3 for each section: $0=$ normal, 1 = mild, 2 = moderate, 3 = severe $(2)$.

\section{Immunochemical Evaluation}

Caspase-3 immunostaining was carried out at room temperature using DAKO Autostainer Universal Staining System (Autostainer Link 48 DAKO, Glostrup, Denmark). First, sections $4 \mu \mathrm{m}$ in thickness obtained from selected paraffin embedded blocks were placed on positively charged slides. Second, all the sections were deparaffinized in xylene and dehydrated through a graded series of ethanol solutions. Third, antigen retrieval was performed at $96^{\circ} \mathrm{C}(10$ $\mathrm{mM} / \mathrm{L}$ citrate buffer, $\mathrm{pH} 6$ ) for 40 minutes in a thermostatic bath (PT Link). The sections were incubated with caspase-3 (3CSP03: sc - 56046; Santa Cruz Biotechnology, USA, 1/100 dilution) for 60 minutes at room temperature. Positive and 
negative controls were added for each antibody and to each batch. A streptavidin-biotin enhanced immunoperoxidase technique (K8000 Envision Flex, DAKO, Glostrup, Denmark) in an automated system was used to show immunoreactions. The sections were incubated with $D A B$ and counterstained lightly with haematoxylin to demonstrate binding. Finally, the sections were dehydrated and mounted with a water based mounting medium. To evaluate the percentage of caspase-3 positive cells, the fibres were scored using the scoring system proposed by Schmeichel et al. (22). Scores ranged from 0 to 4 as follows: $<5 \%, 6-15 \%, 16-25 \%, 26-35 \%$ and $>35 \%$, respectively.

One slide for each case was stained immunohistochemically for nuclear factor kappa B (NF-kB, Santa Cruz Biotechnology, USA, 1/500 dilution). A semi-quantitative grading system was used to evaluate the staining of the antibody, which binds to NF-kB in both axons and Schwann cells; Grade 0: $2 \%$ and below, grade $1: 2 \%-15 \%$, grade $2: 16 \%-25 \%$, grade $3: 26 \%$ $35 \%$, grade $4: 36 \%$, and above (25).

All the sections were examined under a light microscope (Olympus BX52, Olympus Co., Tokyo, Japan). Image Analysis Software (DP-BSW Microscope digital camera software program) was used to assess the samples.

\section{Numbers and Morphological Characteristics of the Axons}

The numbers of axons as well as the morphological characteristics of the axons were evaluated in cross-sections of the sciatic nerve. Five images were taken from each slide. All preparations were counted by taking samples from random areas of each sample using the DP-BSW (Microscope digital camera software) program at a high magnification (100x) under a light microscope (Olympus BX52, Olympus Co., Tokyo, Japan). Counting was performed using the axon counting method previously defined (27). All pathological examinations were evaluated by a pathologist blinded to the experimental group information.

\section{Electrophysiological Evaluation}

Nerve conduction velocity was measured under general anaesthesia (50 and $10 \mathrm{mg} / \mathrm{kg}$, i.p., respectively) with the animals positioned on the left lateral position. After shaving of the animals, the gluteus maximums and biceps femoris muscles were incised to reveal the sciatic nerve. Then, the nerve was dissected and freed from the surrounding tissue and two stimulation electrodes (PY2 50-6873 and PY2 50-1650-Harvard Apparatus, Ltd) were positioned on the sciatic nerve at a $1,1 \mathrm{~cm}$ distance from each other. The sciatic nerves were exposed to supramaximal electrical stimulation (5 $\mathrm{kHz}, 50 \mathrm{~mA}$ ) for $0.2 \mathrm{~ms}$ to stimulate all the fibres of the nerve. Pair of $\mathrm{Ag} / \mathrm{AgCl}$ recording electrodes (EL254S-Biopac System Inc) were placed subcutaneously on the gastrocnemius muscle and a ground electrode was placed between the stimulating and recording electrodes. All measurements were conducted by a Biopac Mp100 System (Biopac Systems Inc., Santa Barbara, CA, USA) and AcqKnowledge Data Acquisition and Analysis Software (v 3.7.2., 1992-2002, Biopac System Inc.). A distal stimulation ( $5 \mathrm{kHz}, 50 \mathrm{~mA}$ for $0.2 \mathrm{~ms}$ ) was given by the stimulating electrode positioned distal to the body and the muscle potential was recorded by the subcutaneous electrodes placed at the gastrocnemius muscle. The same procedure was repeated for the proximal electrode. Nerve conduction velocity was calculated with the measured latency difference. The conduction velocity calculations were made using the following equations:

$\Delta$ Latency: Proximal latency - distal latency (s).

$\Delta$ Distance: Proximal distance - distal distance $(\mathrm{m}): 1 \mathrm{~cm}$.

Nerve conduction velocity: $\Delta$ Distance / $\Delta$ Latency $(\mathrm{m} / \mathrm{s})$.

\section{Statistical Evaluation}

Statistical evaluation of the results was made using the SPSS 22.0 Statistical package (IBM Corp., NY, USA).

The Kolmogorov-Smirnov test was used to determine whether the variables were normally distributed. The Mann-Whitney $U$ test was used to compare the I and T groups and the KruskallWallis $\mathrm{H}$ test was used for the comparison of the $\mathrm{I}, \mathrm{T}$ and $\mathrm{C}$ groups. The Bonferroni corrected post-hoc test was used for pairwise comparisons. Descriptive statistics was used for the variables included the median (25.-75. percentile). $\mathrm{p}<0.05$ was considered as statistically significant.

\section{- RESULTS}

\section{Histopathological Results}

In the transverse sections, centrally placed axons, the surrounding Schwann cells together with the endoneurium were observed. Schwann cells, which envelop the axons, were distinguished by their oval or round nuclei under the endoneurium. Axons were observed to be faded in colour in the cytoplasm of Schwann cells. The presence of a myelin sheath, which is made by Schwann cells and wraps around the axon, was seen in myelinated nerve fibres. Un-myelinated nerve fibres, connective tissue cells and blood vessels were distinguished from the myelinated nerve fibres. In the rats belonging to Group $\mathrm{C}$, and the myelinated area presented an ordered structure and did not show axonal shrinking and nor its swelling (Figure 1A-C).

In rats belonging to groups "I" (Figure 2A, B) and "T" (Figure $3 \mathrm{~A}, \mathrm{~B})$, the axons and myelin sheath presented visible degeneration, the myelin sheath lamellae were separated from each other and the axons were smaller in some nerve fibres, or found to be completely degenerated in other nerve fibres. The presence of degenerated nerve fibres, vacuolization and macrophages, which are responsible for the ingestion of the myelin sheath, were also distinguished in areas with marked degeneration. Axonal degeneration, vacuolization and myelin destruction were found to be markedly greater in Group I than in Group T ( $p=0.037$ ), (Tables I and II).

Fibrotic tissue growth was observed beneath the epineurium surrounding the nerve in the Masson Trichrome preparations of groups "I" (Figure 2C) and "T" (Figure 3C). Masson Trichrome staining was less intense in Group T. 


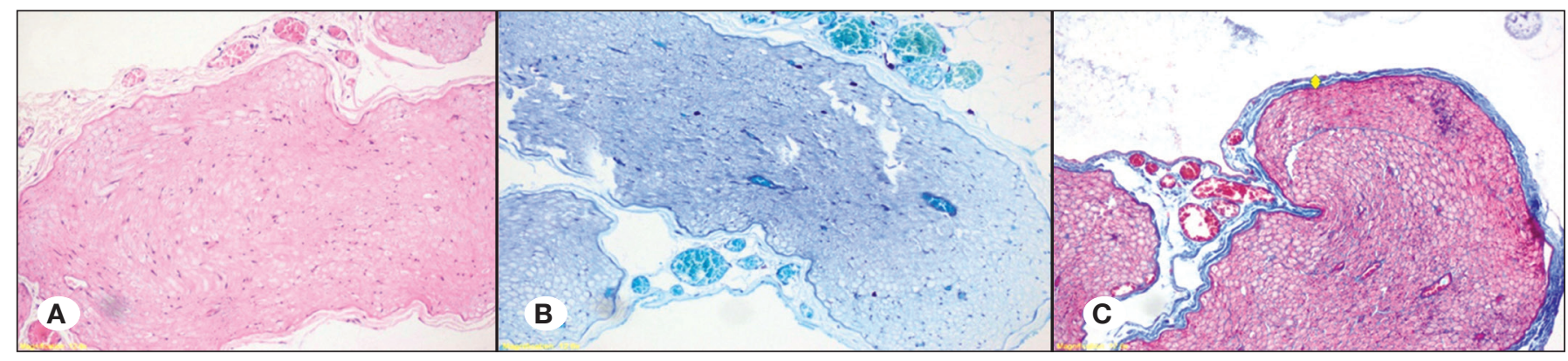

Figure 1: Representative images of the sciatic nerves of rats in Group C (A: Hematoxylin and Eosin staining, 200x magnification; B: Toluidine Blue staining, 200x magnification, C: Masson Trichrom staining, 200x magnification).
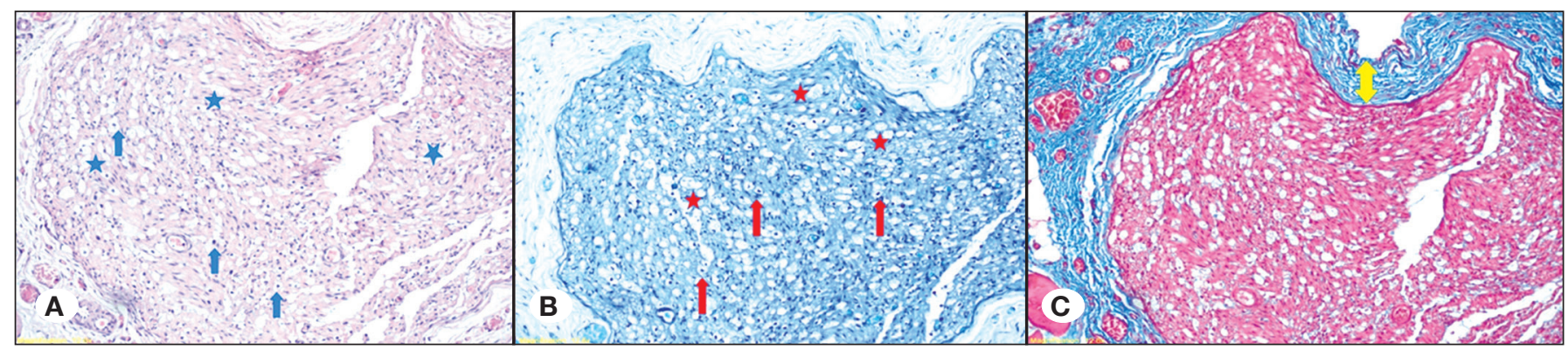

Figure 2: Representative images of the sciatic nerves of rats in Group I Notable features are indicated on the figures as follows: Axonal degeneration and vacuolization (asterisks), Myelin destruction (arrows), Fibrotic tissue growth (yellow arrow) (A: Hematoxylin and Eosin staining, 200x magnification; B: Toluidine Blue staining, 200x magnification, C: Masson Trichrom staining, 200x magnification).
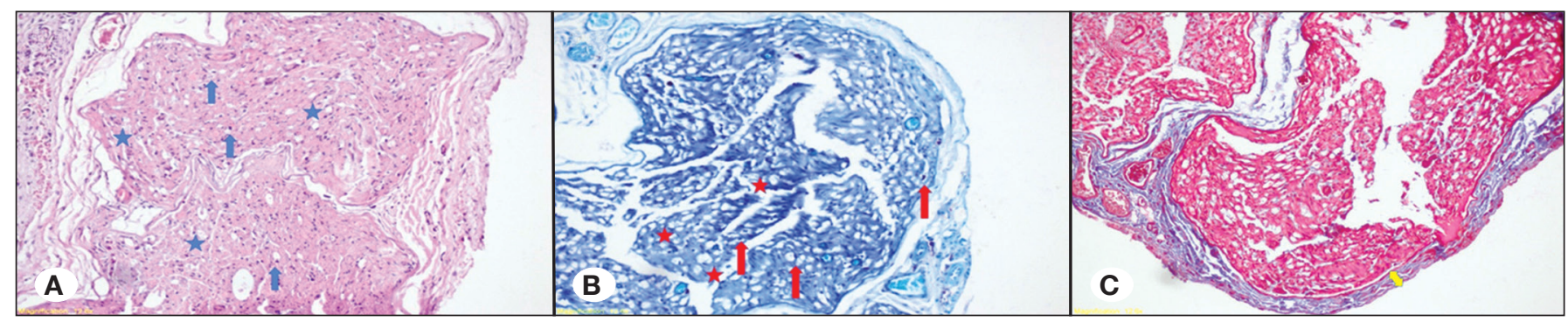

Figure 3: Representative images of the sciatic nerves of rats in Group T Notable features are indicated on the figures as follows: Axonal degeneration and vacuolization (asterisks), Myelin destruction (arrows), Fibrotic tissue growth (yellow arrow) (A: Hematoxylin and Eosin staining, 200x magnification; B: Toluidine Blue staining, 200x magnification, C: Masson Trichrom staining, 200x magnification).

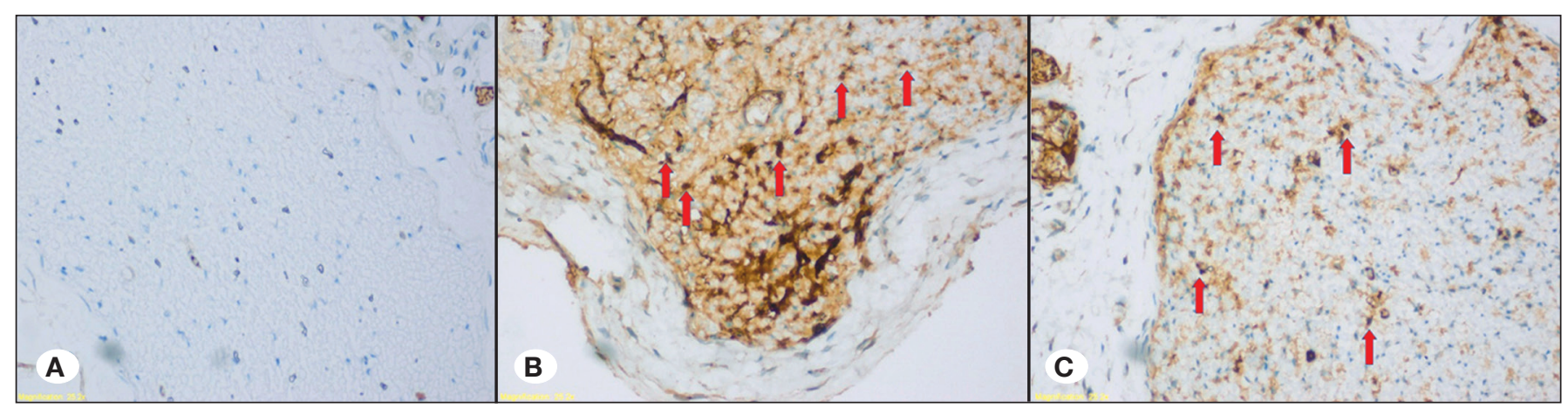

Figure 4: Representative images of Caspase-3 immunohistochemical staining in Groups "C" (A), "I" (B), and "T" (C) (400x magnification). 


\section{Immunochemical Results}

\section{Caspase-3 immunostaining}

Caspase-3 immunostaining was observed in axons and Schwann cells. Stained sections were examined under $600 x$ magnifications. The caspase-3 expression decreased in Group C (Figure 4A). Caspase-3 immunostaining was found to be increased in the preparations of Group I (Figure 4B) and caspase-3 immunoreactivity was observed in the preparations of Group T (Figure 4C), but this staining was less intense than in Group I ( $p=0.053)$.

\section{NF-kB Immunostaining}

NF-kB immunostaining was observed in axons and Schwann cells. No specific staining was observed in Group C (Figure 5A). NF-kB immunostaining was found to be increased in the preparations of Group I (Figure 5B) and NF-kB immunoreactivity was observed in the preparations of Group $\mathrm{T}$ (Figure 5C). Although this staining was less intense than in Group I there were no statistical differences between the "I" and "T" groups $(p=0.184)$.

\section{Number of Axons}

The number of axons was lower in Group I, and the number of axons was significantly higher in Group C $(p<0.001)$. However, there was no statistically significant between Group I and Group T ( $p=0.829)$, (Table III).

\section{Electrophysiological Results}

The proximal and distal latency differences were $0.169 \mathrm{~ms}$ (0.158 ms-0.183 ms), $0.347 \mathrm{~ms}$ (0.310 ms $-0.360 \mathrm{~ms})$, and $0.253 \mathrm{~ms}$ (0.232 ms-0.263 ms) in groups "C", "I", and "T" respectively. There was a statistically significant difference between the "l" and "T" groups $(p<0.034)$. Nerve conductive velocity values were $65.19 \mathrm{~m} / \mathrm{s}(60.07 \mathrm{~m} / \mathrm{s}-69.49 \mathrm{~m} / \mathrm{s}), 31.73$ $\mathrm{m} / \mathrm{s}(30.55 \mathrm{~m} / \mathrm{s}-35.48 \mathrm{~m} / \mathrm{s})$, and $43.42 \mathrm{~m} / \mathrm{s}(41.77 \mathrm{~m} / \mathrm{s}-47.31$ $\mathrm{m} / \mathrm{s})$. There was also a statistically significant difference

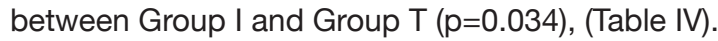

\section{DISCUSSION}

This is the first study to propose a peripheral nerve protective effect of the Tarantula cubensis extract D6. The first of our results was a statistically significant decrease in axon and myelin damage after the application of the Tarantula cubensis extract D6 once a week for four weeks. In addition, this finding was also supported by electrophysiological experiments. Based on our results, our hypothesis that one of the protective effects of the Tarantula cubensis extract D6 may be explained by its anti-inflammatory effects by changing the pro-inflammatory cytokines level, especially TNF- $a$ and IL1. Since IL-1 and TNF-a expression increase after peripheral nerve injury and this state leads to massive neutrophil and macrophage recruitment, damage to the nerve-blood barrier

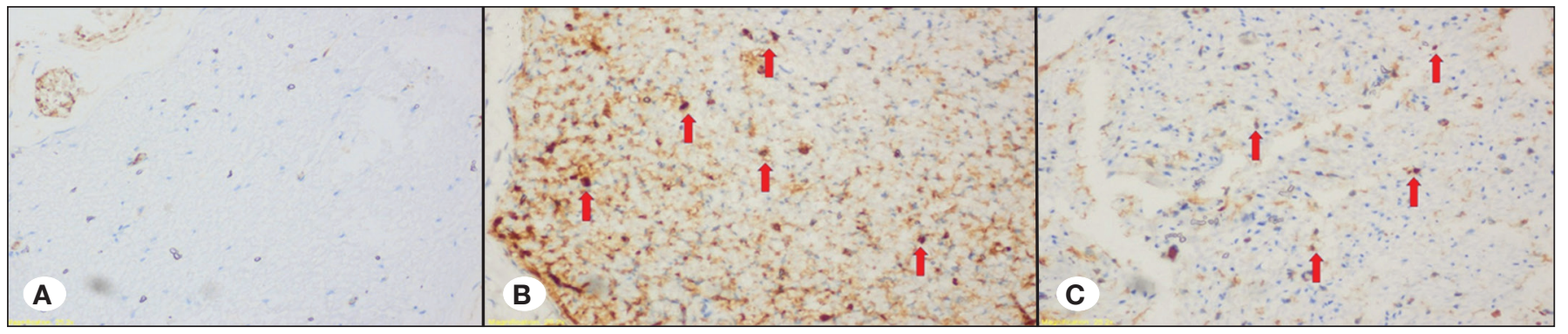

Figure 5: Representative images of NF-kB immunohistochemical staining in groups "C" (A), "I" (B), and "T" (C) (400x magnification).

Table I: Comparison of Results of Histopathological (HP) Examination, Caspase-3 and NF-kB of I and T Groups

\begin{tabular}{lccc}
\hline & $\mathbf{I}(\mathbf{n}=\mathbf{7})$ & $\mathbf{T}(\mathbf{n}=\mathbf{7})$ & $\mathbf{p}$ \\
\hline HP & $3(2-3)$ & $2(2-2)$ & $\mathbf{0 . 0 3 7}$ \\
\hline NF-kB & $2(1-2)$ & $1(1-2)$ & 0.184 \\
\hline Caspase-3 & $3(2-3)$ & $2(1-2)$ & 0.053 \\
\hline
\end{tabular}

Table II: Comparison of Result of Perineurium and Epineurium Thickening, Lymphocytic Infiltration, and Oedema of I and T Groups

\begin{tabular}{llll}
\hline & $\mathbf{I}(\mathbf{n}=\mathbf{7})$ & $\mathbf{T}(\mathbf{n}=7)$ & $\mathbf{p}$ \\
\hline Epineurium thickening & $2(2-3)$ & $2(1-2)$ & 0.097 \\
\hline Perineurium thickening & $2(1-2)$ & $1(1-1)$ & 0.073 \\
\hline Lymphocytic infiltration & $2(1-2)$ & $1(1-2)$ & 0.209 \\
\hline Oedema & $2(1-2)$ & $1(1-2)$ & 0.209 \\
\hline
\end{tabular}

Table III: Comparison of Result of Axon Number of C, I and T Groups

\begin{tabular}{ccccc}
\hline & $\mathbf{C}(\mathbf{n}=7)$ & $\mathbf{I}(\mathbf{n}=\mathbf{7})$ & $\mathbf{T}(\mathbf{n}=\mathbf{7})$ & $\mathbf{p}$ \\
\hline Axon number & $240(237.5-240.5)^{*}$ & $172(165-190)$ & $190(175-202)$ & $\mathbf{0 . 0 0 1}$ \\
\hline
\end{tabular}

${ }^{*}$ Control group is different from others. 
Table IV: Comparison of Electrophysiological Results of C, I and T Groups

\begin{tabular}{lcccc}
\hline & $\mathbf{C ~ ( n = 7 )}$ & $\mathbf{I}(\mathbf{n}=7)$ & $\mathbf{T}(\mathbf{n}=\mathbf{7})$ & $\mathbf{p}$ \\
\hline Latency differences $(\mathrm{ms})$ & $\begin{array}{c}0.169 \\
(0.158-0.183)^{*}\end{array}$ & $\begin{array}{c}0.347 \\
(0.310-0.360)\end{array}$ & $\begin{array}{c}0.253 \\
(0.232-0.263)\end{array}$ & $<0.001$ \\
\hline $\begin{array}{l}\text { Nerve conduction } \\
\text { velocity (m/s) }\end{array}$ & $65.19(60.07-69.49)^{\star}$ & $31.73(30.55-35.48)$ & $43.42(41.77-47.31)$ & $<0.001$ \\
\hline
\end{tabular}

*All groups are statistically different form each other.

and demyelination may occur $(4,5,19)$. Therefore, the increase in IL-1 and TNF-a function or level may cause self-damage. On the other hand, a decrease in IL-1 and TNF-a level in an injured nerve may have a protective effect. In the literature, there are many studies supporting our hypothesis. Also, in the literature, systemic and local application of a TNF-a receptor antagonist have been shown to improve the rate of axonal regeneration (10). In addition, reduction of blood-brain barrier disruption, neutrophil infiltration, IL-6 level and retardation of myelin phagocytosis after administration of IL-1 receptor blocking agents were also shown $(18,19)$.

The second result of our study was that borderline statistical significance of caspase-3 immunochemical staining in Schwann cells was shown. Apoptosis has been defined as a self-destructive programmed biochemical process (28). It has a critical role in the modulation of inflammatory responses and the initiation of healing (26). Apoptosis is frequently mediated by caspase-related proteins in which caspase- 3 plays an important role (28). In previous studies, the potential role of apoptosis and caspase- 3 activation in nerve healing and nerve injury related to neuropathic pain was revealed $(11,24)$. Sekiguchi et al. also showed that distal injury resulted in a greater amount of neuronal apoptosis, enhanced TNF-a expression and caspase-3 levels (23). Therefore, this study revealed the relationship between caspase-3 and TNF-a. In our opinion, this application of the Tarantula cubensis extract D6 may contribute to the survival of the axon and of Schwann cells. Therefore, the Tarantula cubensis D6 extract may contribute to the decrease in level and function of TNF-a after application of the Tarantula cubensis extract D6.

One of the other results of our study which supports our hypothesis was that more fibrotic tissue was observed beneath the epineurium surrounding the nerve in the Masson Trichrome preparations of Group I. This result may also be related to pro-inflammatory cytokine function or level, especially TNF-a, IL-1 and IL-6. Lu et al. showed that TNF-a and IL-1 stimulate fibroblast proliferation to induce neuroma formation (14). In addition, sources of IL-6 in injured peripheral nerves include resident macrophages and fibroblasts (20). Additionally, TNF-a and IL-1 induce the production of IL-6 derived from fibroblasts in injured peripheral nerves (5). Therefore, after the application of the Tarantula cubensis extract D6 may have an anti-fibrotic effect due to the modulation of TNF-a, IL-1 and IL-6 function or level. In addition, in another previous study which was related to the effect of the Tarantula cubensis extract, its anti-fibrotic effect was also shown (17).
Although dosage and application times of the Tarantula cubensis extract are still controversial our opinion is that more than a single application is necessary to observe its anti-inflammatory effects. Our opinion is also supported the literature. Also, a previous study conducted by Corum et al. supports our hypothesis (3). In this study, the authors administered a single subcutaneous dose $(6 \mathrm{ml})$ and then examined pro-inflammatory cytokines such as TNF-a, IL-1 $\beta$, and IL-6. However, they did not show any statistically significant results. In another study conducted by Oryan et al., the Tarantula cubensis extract $(1 \mu \mathrm{g} / \mathrm{kg})$ was administered on days 3,7 , and 10. Macrophages and lymphocyte infiltration were shown to be significantly lower in the injured, Tarantula cubensis extract treated group (17). The first and second experimental studies supports our hypothesis that the Tarantula cubensis extract D6 may have an anti-inflammatory effect which may be related to its application time.

\section{CONCLUSION}

The findings of this study show that the Tarantula cubensis extract D6 decreases axonal and myelin damage after sciatic nerve injury and this neuroprotective effect may be mediated by the altering of pro-inflammatory cytokines levels, especially TNF-a, IL-1 and IL-6. We believe that the Tarantula cubensis extract D6 may be a potential therapeutic agent for peripheral nerve trauma.

\section{REFERENCES}

1. Cam Y, Kibar M, Atasever A, Atalay O, Beyaz L: Efficacy of levamisole and tarantula cubensis venom for the treatment of bovine cutaneous papillomatosis. Vet Rec 160:486-488, 2007

2. Coban YK, Ciralik H, Kurulas EB: Ischemic preconditioning reduces the severity of ischemia-reperfusion injury of peripheral nerve in rats. J Brachial Plex Peripher Nerve Inj 1:2, 2006

3. Corum O, Er A, Dik B: Investigation of the effect of tarantula cubensis extract on acute phase response. Acta Scientiae Veterinariae 44:1414, 2016

4. Dubovy P, Jancalek R, Kubek T: Role of inflammation and cytokines in peripheral nerve regeneration. Int Rev Neurobiol 108:173-206, 2013

5. Fregnan F, Muratori L, Simoes AR, Giacobini-Robecchi MG, Raimondo S: Role of inflammatory cytokines in peripheral nerve injury. Neural Regen Res 7(29):2259-2266, 2012 
6. Gul Satar NY, Cangul IT, Topal A, Kurt H, Ipek V, Onel Gl: The effects of tarantulacubensis venom on open wound healing in rats. J Wound Care 26(2):66-71, 2017

7. Gultekin N, Guvenc T, Kaya D, Agaoglu AR, Ay SS, Kucukaslan I, Emre B, Findik M, Somi-Schafer S, Aslan S: Tarantula cubensis extract alters the degree of apoptosis and mitosis in canine mammary adenocarcinomas. J Vet Sci 16(2):213219,2015

8. Gultiken N, Vural MR: The effect of tarantula cubensis extract applied in pre and postoperative period of canine mammary tumours. JIVS 2:13-23,2007

9. Kaplan AA, Yurt KK, Deniz OG, Altun G: Peripheral nerve and diclofenac sodium. J Chem Neuroanat 87:2-11, 2018

10. Kato K, Liu H, Kikuchi S, Myers RR, Shubayev VI: Immediate antitumor necrosis factor (Etanercept) therapy enhances axonal regeneration after sciatic nerve crush. J Neurosci Res 88(2):360-368, 2010

11. Kim SH, Nam JS, Choi DK, Koh WW, Suh JH, Song JG, Shin JW, Leem JG: Tumor necrosis factor-alpha and apoptosis following spinal nerve ligation injury. Korean J Pain 24(4):185190, 2011

12. Kizilay Z, Aktas S, Kahraman Cetin N, Bakay Ilhan D, Ersoy G, Erken HA: Effect of systemic application bone marrowderived stem cells on healing of peripheral nerve injury in an experimental sciatic nerve injury model. Turk Neurosurg 28(4):654-662, 2018

13. Labroo P, Ho S, Sant H, Shea J, Gale BK, Agarwal J: Controlled delivery of FK506 to improve nerve regeneration. Shock 46(3) Suppl 1:154-159, 2016

14. Lu G, Beuerman RW, Zhao S, Sun G, Nguyen DH, Ma S, Kline DG: Tumour necrosis factor-alpha and interleukin-1 induce activation of MAP kinase and SAP kinase in human neuroma fibroblasts. Neurochem Int 30(4-5):401-410,1997

15. Ma J, Liu J, Yu H, Wang Q, Cheng Y, Xiang L: Curcumin promotes nerve regeneration and functional recovery in rat model of nerve crush injury. Neurosci Lett 547:26-31,2013

16. Nadeau S, Filali M, Zhang J, Kerr BJ, Rivest S, Soulet D, Iwakura Y, de RiveroVaccari JP, Keane RW, Lacroix S: Functional recovery after peripheral nerve injury is dependent on the pro-inflammatory cytokines IL-1 $\beta$, and TNF: Implication of neuropathic pain. J Neurosci 31(35):12533-12542, 2011

17. Oryan A, Moshiri A, Raayat AR: Novel application of theranekron enhanced the structural and functional performance of the tenotomized tendon in rabbits. Cells Tissues Organs 196(5):442-455,2012
18. Perrin FE, Lacroix S, Aviles-Trigueros M, David S: Involvement of monocyte chemoattractant protein-1, macrophage inflammatory protein-1 alpha and interleukin-1 beta in wallerian degeneration. Brain 128(Pt4):854-866, 2005

19. Pradillo JM, Denes A, Greenhalgh AD, Boutin H, Drake C, McColl BW, Barton E, Proctor SD, Russell JC, Rothwell NJ, Allan SM: Delayed administration of interleukin-1 receptor antagonist reduces ischemic brain damage and inflammation in comorbid rats. J Cereb Blood Flow Metab 32(9):1810-1819, 2012

20. Reichert F, Levitzky R, Rotshenker S: Interleukin 6 intact and injured mouse peripheral nerves. Eur J Neurosci 8(3):530-535, 1996

21. Sardari K, Kakhhi EG, Mohri M: Evaluation of wound contraction and epithelialization after subcutaneous administration of theranekron in cows. Comp Clin Pathol 16:197-200, 2007

22. Schmeichel AM, Schmelzer JD, Low PA: Oxidative injury and apoptosis of dorsal root ganglion neurons in chronic experimental diabetic neuropathy. Diabetes 52(1):165-171, 2003

23. Sekiguchi M, Sekiguchi Y, Konno S, Kobayashi H, Homma Y, Kikuchi S: Comparison of neuropathic pain and neuronal apoptosis following nerve root and spinal nerve compression. Eur Spine J 18(12):1978-1985, 2009

24. Sorkin LS, Doom CM: Epineuronal application of TNF elicits an acute mechanical hyperalgesia in the awake rat. J Peripher Nerv Syst 5(2):96-100, 2000

25. Wang Y, Schmeichel AM, Lida H, Schmelzer JD, Low PA: Enhanced inflammatory response via activation of NF-kappa $B$ in acute experimental diabetic neuropathy subjected to ischemia-reperfusion injury. J Neurol Sci 247:47-52,2006

26. Wu YS, Chen SN: Apoptotic cell: Linkage of inflammation and wound healing. Front Pharmacol 5:1,2014

27. Yang I, Bashaw GJ: Son of sevenless directly links the roborecep- tor to rac activation to control axon repulsion at the midline. Neuron 52:595-607,2006

28. Yuksel TN, Halici Z, Demir R, Cakir M, Calikoglu C, Ozdemir G, Unal D: Investigation of the effect of telmisartan on experimental induced peripheral nerve injury in rats. Int $J$ Neurosci 125(6):464-473, 2015 